\title{
Existence of nonoscillatory solutions for fractional neutral functional differential equation
}

\author{
Velu Muthulakshmi ${ }^{1 *}$ and Subramani Pavithra ${ }^{2}$
}

\begin{abstract}
In this paper, we study the existence of nonoscillatory solutions for a kind of fractional neutral functional differential equation with Liouville fractional derivative of order $\alpha \geq 0$ on the half-axis. Some sufficient conditions are established using Krasnoselskii's and Schauder's fixed point theorems.
\end{abstract}

\section{Keywords}

Nonoscillatory solution, Liouville fractional derivative, neutral equation.

\section{AMS Subject Classification}

34C10, 34K11, 34A08.

1,2 Department of Mathematics, Periyar University, Salem, Tamil Nadu-636011, India.

*Corresponding author: ${ }^{\text {* }}$ vmuthupu@gmail.com; ${ }^{2}$ pavithrasubramani51@gmail.com

Article History: Received 19 October 2019; Accepted 17 December 2019

\section{Contents}

1 Introduction 12

2 Preliminaries 13

3

Main Results .13

References 18

\section{Introduction}

Fractional calculus is a subject involving non-integer order derivatives and integrals. It has many applications in different fields such as physics, chemistry, mechanics, engineering etc. There have been many books on fractional differential equations, we refer the reader to $[7,8,10]$ and the references cited therein. Many authors have studied some aspects of fractional differential equations, such as the existence and uniqueness of solutions to Cauchy type problems and the stability of solutions.

Oscillation theory is one of the most important qualitative properties of solutions of differential equations. Oscillatory behavior of ordinary differential equations can be found in the monographs [1-3] and the references cited therein. But, in recent years, oscillation theory of fractional differential equations have been developed rapidly, see [4-6, 9, 11, 12].

Recently, in [11], Zhou et al. discussed the existence of nonoscillatory solutions of the following fractional differential equation

$$
D_{t}^{\alpha}[x(t)-c x(t-\tau)]+\sum_{i=1}^{m} P_{i}(t) x\left(t-\sigma_{i}\right)=0, t \geq t_{0},
$$

where $D_{t}^{\alpha}$ denotes the Liouville fractional derivative of order $\alpha \in[1,+\infty)$ on the half-axis.

In [12], Zhou et al. studied the existence of nonoscillatory solutions of

$D_{t}^{\alpha}[x(t)+c x(t-\tau)]^{\prime}+\sum_{i=1}^{m} P_{i}(t) F_{i}\left(x\left(t-\sigma_{i}\right)\right)=0, t \geq t_{0}$,

where $D_{t}^{\alpha}$ denotes the Liouville fractional derivatives of order $\alpha \geq 0$ on the half-axis.

Motivated by the above literature, in this paper, we establish some sufficient conditions for the existence of nonoscillatory solutions of neutral functional differential equation of fractional order

$$
\begin{aligned}
& \left(r(t) D_{t}^{\alpha}\left[x(t)+C(t) x\left(t-\tau_{1}\right)\right]^{\prime}\right)^{\prime} \\
& \quad+\sum_{i=1}^{m} P_{i}(t) F_{i}\left(x\left(t-\sigma_{i}\right)\right)=g(t), t \geq t_{0},
\end{aligned}
$$

where $D_{-}^{\alpha}$ is Liouville fractional derivative of order $\alpha \geq 0$ on the half-axis, $C, P_{i}, g \in C\left(\left[t_{0}, \infty\right), \mathbb{R}\right), r \in C\left(\left[t_{0}, \infty\right), \mathbb{R}^{+}\right), F_{i} \in$ $C(\mathbb{R}, \mathbb{R}), \tau, \sigma_{i} \in \mathbb{R}^{+}$for $i=1,2, \ldots, m, m \geq 1$ is an integer.

Let $l=\max _{1 \leq i \leq m}\left\{\tau, \sigma_{i}\right\}$. By a solution of Eq.(1.1), we mean a function $x \in C\left(\left[t_{1}-l, \infty\right), \mathbb{R}\right)$ for some $t_{1} \geq t_{0}$ such that $\left(r(t) D_{-}^{\alpha}\left[x(t)+C_{1}(t) x\left(t-\tau_{1}\right)\right]^{\prime}\right)^{\prime}$ exists on $\left[t_{1}, \infty\right)$ and Eq.(1.1) 
is satisfied for $t \geq t_{1}$.

A nontrivial solution of Eq.(1.1) is called oscillatory if it has arbitrarily large zeros, otherwise it is called nonoscillatory. Equation (1.1) is called oscillatory if all of its solutions are oscillatory.

In this paper, we obtain some sufficient conditions for the existence of nonoscillatory solution of Eq.(1.1) by using Krasnosel'skii's and Schauder's fixed point theorems.

\section{Preliminaries}

In this section, we provide some preliminary details which will be used in the subsequent section.

Definition 2.1. [7] The Liouville fractional integral on the half-axis is defined by

$$
D_{t}^{-\alpha} f(t)=\frac{1}{\Gamma(\alpha)} \int_{t}^{\infty}(s-t)^{\alpha-1} f(s) d s
$$

where $t \in \mathbb{R}$ and $\alpha \in[0, \infty)$.

Definition 2.2. [7] The Liouville fractional derivative on the half-axis is defined by

$D_{t}^{\alpha} f(t)=\frac{1}{\Gamma(n-\alpha)}(-1)^{n} \frac{d^{n}}{d t^{n}}\left(\int_{t}^{\infty}(s-t)^{n-\alpha-1} f(s) d s\right)$,

where $n=[\alpha]+1, \alpha \in(0, \infty),[\alpha]$ denotes the integer part of $\alpha$ and $t \in \mathbb{R}$.

In particular, if $\alpha=n \in \mathbb{N}$, then $D_{t}^{n} f(t)=(-1)^{n} f^{(n)}(t)$ and it has the property $D_{t}^{\alpha}\left(D_{t}^{-\alpha} f(t)\right)=f(t)$ for $\alpha>0$.

Lemma 2.3. [1] (Arzela-Ascoli theorem) A subset $E$ in $C([a, b], \mathbb{R})$ with norm $\|f\|=\sup _{t \in[a, b]}|f(t)|$ is relatively compact if and only if it is uniformly bounded and equicontinuous on $[a, b]$.

Lemma 2.4. [2] (Krasnosel'skii's fixed point theorem) Suppose $X$ is a Banach space and $\Omega$ is a bounded, convex and closed subset of $X$. Let $T_{1}, T_{2}: \Omega \rightarrow X$ satisfy the following conditions:

(i) $T_{1} x+T_{2} y \in \Omega$ for any $x, y \in \Omega$,

(ii) $T_{1}$ is a contraction mapping,

(iii) $T_{2}$ is completely continuous.

Then $T_{1}+T_{2}$ has a fixed point in $\Omega$, i.e., the equation $T_{1} x+$ $T_{2} x=x$ has a solution in $\Omega$.

Lemma 2.5. [2] (Schauder's fixed point theorem) Let $\Omega$ be a closed, convex and nonempty subset of a Banach space $X$. Let $T: \Omega \rightarrow \Omega$ be a continuous mapping such that $T \Omega$ is a relatively compact subset of $X$. Then $T$ has at least one fixed point in $\Omega$. That is, there exists an $x \in \Omega$ such that $T x=x$.

\section{Main Results}

In this section, some sufficient conditions are established for the existence of nonoscillatory solutions of Eq.(1.1) for the following cases:

$$
\begin{aligned}
& -1<c_{1} \leq C(t) \leq 0,-\infty<C(t) \leq c_{2}<-1, \\
& 0 \leq C(t) \leq c_{3}<1,1<c_{4} \leq C(t)<\infty \text { and } C(t) \equiv-1 .
\end{aligned}
$$

Theorem 3.1. Assume that $-1<c_{1} \leq C(t) \leq 0$ and that

$$
\int_{t_{0}}^{\infty} \int_{t_{0}}^{s} \frac{s^{\alpha}}{r(s)}\left|P_{i}(u)\right| d u d s<\infty, i=1,2, \ldots, m
$$

and

$$
\int_{t_{0}}^{\infty} \int_{t_{0}}^{s} \frac{s^{\alpha}}{r(s)}|g(u)| d u d s<\infty
$$

Then Eq.(1.1) has a bounded nonoscillatory solution.

Proof. Because of conditions (3.1) and (3.2), we can choose a $t_{1}>t_{0}$ sufficiently large such that

$$
\begin{aligned}
& \frac{1}{\Gamma(\alpha+1)} \int_{t}^{\infty} \frac{(s-t)^{\alpha}}{r(s)} \\
& \times \int_{t_{1}}^{s}\left(\sum_{i=1}^{m}\left|P_{i}(u)\right| M_{1}+|g(u)|\right) d u d s \leq \frac{1+c_{1}}{3},
\end{aligned}
$$

where $M_{1}=\max _{\frac{2\left(1+c_{1}\right)}{3} \leq x \leq \frac{4}{3}}\left\{\left|F_{i}(f x)\right|: 1 \leq i \leq m\right\}$.

Let $C\left(\left[t_{0}, \infty\right), \mathbb{R}\right)$ be the set of all continuous functions with the norm $\|x\|=\sup _{t \geq t_{0}}|x(t)|<\infty$. Then $C\left(\left[t_{0}, \infty\right), \mathbb{R}\right)$ is a Banach space. We define a closed, bounded and convex subset $\Omega$ of $C\left(\left[t_{0}, \infty\right), \mathbb{R}\right)$ as follows:

$\Omega=\left\{x=x(t) \in C\left(\left[t_{0}, \infty\right), \mathbb{R}\right): \frac{2\left(1+c_{1}\right)}{3} \leq x \leq \frac{4}{3}, t \geq t_{0}\right\}$.

Define two maps $T_{1}$ and $T_{2}: \Omega \rightarrow C\left(\left[t_{0}, \infty\right), \mathbb{R}\right)$ as follows:

$$
\left(T_{1} x\right)(t)= \begin{cases}1+c_{1}-C(t) x\left(t-\tau_{1}\right), & t \geq t_{1}, \\ \left(T_{1} x\right)\left(t_{1}\right), & t_{0} \leq t \leq t_{1}\end{cases}
$$

$$
\left(T_{2} x\right)(t)= \begin{cases}\frac{1}{\Gamma(\alpha+1)} \int_{t}^{\infty} \frac{(s-t)^{\alpha}}{r(s)} & \\ \times \int_{t_{1}}^{s}\left(\sum_{i=1}^{m} P_{i}(u) F_{i}\left(x\left(u-\sigma_{i}\right)\right)\right. & \\ \quad-g(u)) d u d s, & t \geq t_{1}, \\ \left(T_{2} x\right)\left(t_{1}\right), & t_{0} \leq t \leq t_{1} .\end{cases}
$$


For every $x, y \in \Omega$ and $t \geq t_{1}$, we get

$$
\begin{aligned}
\left(T_{1} x\right)(t)+ & \left(T_{2} y\right)(t) \\
\leq & 1+c_{1}-C(t) x(t-\tau)+\frac{1}{\Gamma(\alpha+1)} \int_{t}^{\infty} \frac{(s-t)^{\alpha}}{r(s)} \\
& \times \int_{t_{1}}^{s}\left(\sum_{i=1}^{m}\left|P_{i}(u)\right|\left|F_{i}\left(x\left(u-\sigma_{i}\right)\right)\right|+|g(u)|\right) d u d s \\
\leq & 1+c_{1}-\frac{4}{3} c_{1}+\frac{1}{\Gamma(\alpha+1)} \int_{t}^{\infty} \frac{(s-t)^{\alpha}}{r(s)} \\
& \times \int_{t_{1}}^{s}\left(\sum_{i=1}^{m}\left|P_{i}(u)\right| M_{1}+|g(u)|\right) d u d s \\
\leq & 1+c_{1}-\frac{4}{3} c_{1}+\frac{1+c_{1}}{3}=\frac{4}{3}
\end{aligned}
$$

and

$$
\begin{aligned}
\left(T_{1} x\right)(t)+ & \left(T_{2} y\right)(t) \\
\geq & 1+c_{1}-C(t) x(t-\tau)-\frac{1}{\Gamma(\alpha+1)} \int_{t}^{\infty} \frac{(s-t)^{\alpha}}{r(s)} \\
& \times \int_{t_{1}}^{s}\left(\sum_{i=1}^{m}\left|P_{i}(u)\right|\left|F_{i}\left(x\left(u-\sigma_{i}\right)\right)\right|+|g(u)|\right) d u d s \\
\geq & 1+c_{1}-\frac{1}{\Gamma(\alpha+1)} \int_{t}^{\infty} \frac{(s-t)^{\alpha}}{r(s)} \\
& \times \int_{t_{1}}^{s}\left(\sum_{i=1}^{m}\left|P_{i}(u)\right| M_{1}+|g(u)|\right) d u d s \\
\geq & 1+c_{1}-\frac{1+c_{1}}{3}=\frac{2\left(1+c_{1}\right)}{3} .
\end{aligned}
$$

Thus, $\left(T_{1} x\right)(t)+\left(T_{2} y\right)(t) \in \Omega$ for any $x, y \in \Omega$. Now, for $x, y \in$ $\Omega$ and $t \geq t_{1}$, we have

$$
\begin{aligned}
\left|\left(T_{1} x\right)(t)-\left(T_{1} y\right)(t)\right| & \leq-C(t)|x(t-\tau)-y(t-\tau)| \\
& \leq-c_{1}\|x-y\| .
\end{aligned}
$$

This implies with the supremum norm that

$$
\left\|\left(T_{1} x\right)(t)-\left(T_{2} y\right)(t)\right\| \leq-c_{1}\|x-y\| .
$$

Since $0<-c_{1}<1$, which shows that $T_{1}$ is a contraction mapping on $\Omega$. Next, we have to show that $T_{2}$ is completely continuous. It is enough to show that $T_{2}$ is continuous and $T_{2} \Omega$ is relatively compact.

First, we will show that $T_{2}$ is continuous. Let $x_{k}(t)$ be a sequence in $\Omega$ such that $x_{k}(t) \rightarrow x(t)$ as $k \rightarrow \infty$. Since $\Omega$ is closed, $x=x(t) \in \Omega$. For $t \geq t_{1}$, we have

$$
\begin{aligned}
& \left|\left(T_{2} x_{k}\right)(t)-\left(T_{2} x\right)(t)\right| \\
& \leq \frac{1}{\Gamma(\alpha+1)} \int_{t}^{\infty} \frac{(s-t)^{\alpha}}{r(s)} \\
& \quad \times \int_{t_{1}}^{s}\left(\sum_{i=1}^{m}\left|P_{i}(u)\right|\left|F_{i}\left(x_{k}\left(s-\sigma_{i}\right)\right)-F_{i}\left(x\left(s-\sigma_{i}\right)\right)\right|\right) d u d s
\end{aligned}
$$

Since $\left|F_{i}\left(x_{k}\left(t-\sigma_{i}\right)\right)-F_{i}\left(x\left(t-\sigma_{i}\right)\right)\right| \rightarrow 0$ as $k \rightarrow \infty$ for $i=$ $1,2, \ldots, m$, by Lebesgue-dominated convergence theorem, we get that $\lim _{t \rightarrow \infty}\left\|\left(T_{2} x_{k}\right)(t)-\left(T_{2} x\right)(t)\right\|=0$. Thus, $T_{2}$ is continuous.

In order to show that $T_{2} \Omega$ is relatively compact, it is enough to show that the family of functions $\left\{T_{2} x: x \in \Omega\right\}$ is uniformly bounded and equicontinuous on $\left[t_{0}, \infty\right)$. The uniformly boundedness is obvious. For the equicontinuity, by Levitan's result [3], it is enough to show that, for any given $\varepsilon>0,\left[t_{1}, \infty\right)$ can be decomposed into finite subintervals in such a way that all functions of the family have oscillation less than $\varepsilon$ on each subinterval. By (3.1) and (3.2), for any $\varepsilon>0$, choose $T \geq t_{1}$ sufficiently large such that

$\frac{1}{\Gamma(\alpha+1)} \int_{T}^{\infty} \frac{(s-t)^{\alpha}}{r(s)} \int_{t_{1}}^{s}\left(\sum_{i=1}^{m}\left|P_{i}(u)\right| M_{1}+|g(u)|\right) d u d s<\frac{\varepsilon}{2}$.

For $t_{3}>t_{2} \geq T$ and $x \in \Omega$, we obtain

$$
\begin{aligned}
\mid\left(T_{2} x\right)\left(t_{3}\right) & -\left(T_{2} x\right)\left(t_{2}\right) \mid \\
\leq & \frac{1}{\Gamma(\alpha+1)} \int_{t_{3}}^{\infty} \frac{(s-t)^{\alpha}}{r(s)} \\
& \times \int_{t_{1}}^{s}\left(\sum_{i=1}^{m}\left|P_{i}(u)\right|\left|F_{i}\left(x\left(s-\sigma_{i}\right)\right)\right|+|g(u)|\right) d u d s \\
& +\frac{1}{\Gamma(\alpha+1)} \int_{t_{2}}^{\infty} \frac{(s-t)^{\alpha}}{r(s)} \\
& \times \int_{t_{1}}^{s}\left(\sum_{i=1}^{m}\left|P_{i}(u)\right|\left|F_{i}\left(x\left(s-\sigma_{i}\right)\right)\right|+|g(u)|\right) d u d s \\
\leq & \frac{1}{\Gamma(\alpha+1)} \int_{t_{3}}^{\infty} \frac{(s-t)^{\alpha}}{r(s)} \\
& \times \int_{t_{1}}^{s}\left(\sum_{i=1}^{m}\left|P_{i}(u)\right| M_{1}+|g(u)|\right) d u d s \\
& +\frac{1}{\Gamma(\alpha+1)} \int_{t_{2}}^{\infty} \frac{(s-t)^{\alpha}}{r(s)} \\
& \times \int_{t_{1}}^{s}\left(\sum_{i=1}^{m}\left|P_{i}(u)\right| M_{1}+|g(u)|\right) d u d s \\
< & \frac{\varepsilon}{2}+\frac{\varepsilon}{2}=\varepsilon .
\end{aligned}
$$

For $t_{1} \leq t_{2}<t_{3} \leq T$ and $x \in \Omega$, we have

$$
\begin{aligned}
& \left|\left(T_{2} x\right)\left(t_{3}\right)-\left(T_{1} x\right)\left(t_{2}\right)\right| \\
& \leq \frac{1}{\Gamma(\alpha+1)} \int_{t_{2}}^{t_{3}} \frac{(s-t)^{\alpha}}{r(s)} \\
& \quad \times \int_{t_{1}}^{s}\left(\sum_{i=1}^{m}\left|P_{i}(u)\right|\left|F_{i}\left(x\left(s-\sigma_{i}\right)\right)\right|+|g(u)|\right) d u d s \\
& \leq \frac{1}{\Gamma(\alpha+1)} \int_{t_{2}}^{t_{3}} \frac{(s-t)^{\alpha}}{r(s)} \\
& \quad \times \int_{t_{1}}^{s}\left(\sum_{i=1}^{m}\left|P_{i}(u)\right| M_{1}+|g(u)|\right) d u d s
\end{aligned}
$$




$$
\begin{aligned}
\leq & \frac{1}{\Gamma(\alpha+1)} \max _{t_{1} \leq s \leq T}\left\{\frac{(s-t)^{\alpha}}{r(s)}\right. \\
& \left.\times \int_{t_{1}}^{s}\left(\sum_{i=1}^{m}\left|P_{i}(u)\right| M_{1}+|g(u)|\right) d u\right\}\left(t_{3}-t_{2}\right),
\end{aligned}
$$

and hence there exists a $\delta>0$ such that

$$
\left|\left(T_{2} x\right)\left(t_{3}\right)-\left(T_{2} x\right)\left(t_{2}\right)\right|<\varepsilon, \text { whenever } 0<t_{3}-t_{2}<\delta
$$

Also, for $t_{0} \leq t_{2}<t_{3} \leq t_{1}$ and $x \in \Omega$, we can easily see that

$$
\left|\left(T_{2} x\right)\left(t_{3}\right)-\left(T_{2} x\right)\left(t_{2}\right)\right|=0<\varepsilon .
$$

Therefore, $\left\{T_{2} x: x \in \Omega\right\}$ is equicontinuous on $\left[t_{0}, \infty\right)$ and hence $T_{2} \Omega$ is relatively compact. Thus, by Krasnosel'skii's fixed point theorem, $T_{1} x+T_{2} x=x$ has a solution in $\Omega$, which is a bounded positive solution of Eq.(1.1). Hence the proof is complete.

Theorem 3.2. Assume that $-\infty<C(t) \equiv c_{2}<-1$ and that conditions (3.1) and (3.2) hold. Then Eq.(1.1) has a bounded nonoscillatory solution.

Proof. Because of conditions (3.1) and (3.2), we can choose a $t_{1}>t_{0}$,

$$
t_{1}+\tau \geq t_{0}+\max \left\{\sigma_{i}\right\}
$$

sufficiently large such that

$$
\begin{aligned}
& \frac{1}{c_{2} \Gamma(\alpha+1)} \int_{t+\tau}^{\infty} \frac{(s-t-\tau)^{\alpha}}{r(s)} \\
& \times \int_{t_{1}+\tau}^{s}\left(\sum_{i=1}^{m}\left|P_{i}(u)\right| M_{2}+|g(u)|\right) d u d s \leq \frac{-c_{2}+1}{2},
\end{aligned}
$$

where $M_{2}=\max _{\frac{-\left(c_{2}+1\right)}{2} \leq x \leq-2 c_{2}}\left\{\left|F_{i}(x)\right|: 1 \leq i \leq m\right\}$.

Let $C\left(\left[t_{0}, \infty\right), \mathbb{R}\right)$ be the set of all continuous functions with the norm $\|x\|=\sup _{t \geq t_{0}}|x(t)|<\infty$. Then $C\left(\left[t_{0}, \infty\right), \mathbb{R}\right)$ is a Banach space. We define a closed, bounded and convex subset $\Omega$ of $C\left(\left[t_{0}, \infty\right), \mathbb{R}\right)$ as follows:

$$
\Omega=\left\{\begin{array}{c}
x=x(t) \in C\left(\left[t_{0}, \infty\right), \mathbb{R}\right):-\frac{\left(c_{2}+1\right)}{2} \leq x \leq-2 c_{2}, \\
t \geq t_{0}
\end{array}\right\} .
$$

Define two maps $T_{1}$ and $T_{2}: \Omega \rightarrow C\left(\left[t_{0}, \infty\right), \mathbb{R}\right)$ as follows:

$$
\begin{gathered}
\left(T_{1} x\right)(t)= \begin{cases}-c_{2}-1-\frac{1}{C(t)} x(t+\tau), & t \geq t_{1}, \\
\left(T_{1} x\right)\left(t_{1}\right), & t_{0} \leq t \leq t_{1},\end{cases} \\
\left(T_{2} x\right)(t)= \begin{cases}\frac{1}{C(t) \Gamma(\alpha+1)} \int_{t+\tau}^{\infty} \frac{(s-t-\tau)^{\alpha}}{r(s)} & t \geq t_{1}, \\
\times \int_{t_{1}+\tau}^{s}\left(\sum_{i=1}^{m} P_{i}(u) F_{i}\left(x\left(u-\sigma_{i}\right)\right)\right. & t_{0} \leq t \leq t_{1} . \\
\left(T_{2} x\right)\left(t_{1}\right), & \end{cases}
\end{gathered}
$$

For every $x, y \in \Omega$ and $t \geq t_{1}$, we get

$$
\begin{aligned}
\left(T_{1} x\right)(t) & +\left(T_{2} y\right)(t) \\
\leq \quad & -c_{2}-1-\frac{1}{C(t)} x(t+\tau) \\
& -\frac{1}{C(t) \Gamma(\alpha+1)} \int_{t+\tau}^{\infty} \frac{(s-t-\tau)^{\alpha}}{r(s)} \\
& \times \int_{t_{1}+\tau}^{s}\left(\sum_{i=1}^{m}\left|P_{i}(u)\right|\left|F_{i}\left(x\left(u-\sigma_{i}\right)\right)\right|+|g(u)|\right) d u d s \\
\leq \quad & -c_{2}-1+2-\frac{1}{c_{2} \Gamma(\alpha+1)} \int_{t+\tau}^{\infty} \frac{(s-t-\tau)^{\alpha}}{r(s)} \\
\quad & \times \int_{t_{1}+\tau}^{s}\left(\sum_{i=1}^{m}\left|P_{i}(u)\right| M_{2}+|g(u)|\right) d u d s \\
\leq \quad & -c_{2}+1-\frac{c_{2}+1}{2}=-2 c_{2}
\end{aligned}
$$

and

$$
\begin{aligned}
\left(T_{1} x\right)(t) & +\left(T_{2} y\right)(t) \\
\geq & -c_{2}-1-\frac{1}{C(t)} x(t+\tau) \\
& +\frac{1}{C(t) \Gamma(\alpha+1)} \int_{t+\tau}^{\infty} \frac{(s-t-\tau)^{\alpha}}{r(s)} \\
& \times \int_{t_{1}+\tau}^{s}\left(\sum_{i=1}^{m}\left|P_{i}(u)\right|\left|F_{i}\left(x\left(u-\sigma_{i}\right)\right)\right|+|g(u)|\right) d u d s \\
\geq & -c_{2}-1+\frac{1}{c_{2} \Gamma(\alpha+1)} \int_{t+\tau}^{\infty} \frac{(s-t-\tau)^{\alpha}}{r(s)} \\
& \times \int_{t_{1}+\tau}^{s}\left(\sum_{i=1}^{m}\left|P_{i}(u)\right| M_{2}+|g(u)|\right) d u d s \\
\geq \quad & -c_{2}-1+\frac{c_{2}+1}{2} \\
= & -\frac{c_{2}+1}{2} .
\end{aligned}
$$

Thus, $\left(T_{1} x\right)(t)+\left(T_{2} y\right)(t) \in \Omega$ for any $x, y \in \Omega$. Now, for $x, y \in \Omega$ and $t \geq t_{1}$, we have

$$
\begin{aligned}
\left|\left(T_{1} x\right)(t)-\left(T_{1} y\right)(t)\right| & \leq-\frac{1}{C(t)}|x(t-\tau)-y(t-\tau)| \\
& \leq-\frac{1}{c_{2}}\|x-y\| .
\end{aligned}
$$

This implies with the supremum norm that

$$
\left\|\left(T_{1} x\right)(t)-\left(T_{2} y\right)(t)\right\| \leq-\frac{1}{c_{2}}\|x-y\| .
$$

Since $0<-\frac{1}{c_{2}}<1$, which shows that $T_{1}$ is a contraction mapping on $\Omega$.

Proceeding similarly as in the proof of Theorem 3.1, we obtain that the mapping $T_{2}$ is completely continuous. Thus, by Krasnosel'skii's fixed point theorem, $T_{1} x+T_{2} x=x$ has a solution in $\Omega$, which is a bounded positive solution of Eq.(1.1). Hence the proof is complete. 
Theorem 3.3. Assume that $0 \leq C(t) \leq c_{3}<1$ and that conditions (3.1) and (3.2) hold. Then Eq.(1.1) has a bounded nonoscillatory solution.

Proof. Because of (3.1) and (3.2), we can choose a $t_{1}>t_{0}$ sufficiently large such that

$$
\begin{aligned}
& \frac{1}{\Gamma(\alpha+1)} \int_{t}^{\infty} \frac{(s-t)^{\alpha}}{r(s)} \\
& \quad \times \int_{t_{1}}^{s}\left(\sum_{i=1}^{m}\left|P_{i}(u)\right| M_{3}+|g(u)|\right) d u d s \leq 1-c_{3},
\end{aligned}
$$

where $M_{3}=\max _{2\left(1-c_{3}\right) \leq x \leq 4}\left\{\left|F_{i}(x)\right|: 1 \leq i \leq m\right\}$.

Let $C\left(\left[t_{0}, \infty\right), \mathbb{R}\right)$ be the set of all continuous functions with the norm $\|x\|=\sup |x(t)|<\infty$. Then $C\left(\left[t_{0}, \infty\right), \mathbb{R}\right)$ is a Banach space. We define a closed, bounded and convex subset $\Omega$ of $C\left(\left[t_{0}, \infty\right), \mathbb{R}\right)$ as follows:

$\Omega=\left\{x=x(t) \in C\left(\left[t_{0}, \infty\right), \mathbb{R}\right): 2\left(1-c_{3}\right) \leq x \leq 4, t \geq t_{0}\right\}$.

Define two maps $T_{1}$ ans $T_{2}: \Omega \rightarrow C\left(\left[t_{0}, \infty\right), \mathbb{R}\right)$ as follows:

$$
\begin{gathered}
\left(T_{1} x\right)(t)= \begin{cases}3+c_{3}-C(t) x\left(t-\tau_{1}\right), & t \geq t_{1}, \\
\left(T_{1} x\right)\left(t_{1}\right), & t_{0} \leq t \leq t_{1},\end{cases} \\
\left(T_{2} x\right)(t)= \begin{cases}\frac{1}{\Gamma(\alpha+1)} \int_{t}^{\infty} \frac{(s-t)^{\alpha}}{r(s)} \\
\times \int_{t_{1}}^{s}\left(\sum_{i=1}^{m} P_{i}(u) F_{i}\left(x\left(u-\sigma_{i}\right)\right)\right. \\
-g(u)) d u d s, & t \geq t_{1}, \\
\left(T_{2} x\right)\left(t_{1}\right), & t_{0} \leq t \leq t_{1} .\end{cases}
\end{gathered}
$$

For every $x, y \in \Omega$ and $t \geq t_{1}$, we get

$$
\begin{aligned}
\left(T_{1} x\right)(t)+ & \left(T_{2} y\right)(t) \\
\leq & 3+c_{3}-C(t) x(t-\tau) \\
& +\frac{1}{\Gamma(\alpha+1)} \int_{t}^{\infty} \frac{(s-t)^{\alpha}}{r(s)} \\
& \int_{t_{1}}^{s}\left(\sum_{i=1}^{m}\left|P_{i}(u)\right|\left|F_{i}\left(x\left(u-\sigma_{i}\right)\right)\right|\right. \\
& +|g(u)|) d u d s \\
\leq \quad & 3+c_{3}+\frac{1}{\Gamma(\alpha+1)} \int_{t}^{\infty} \frac{(s-t)^{\alpha}}{r(s)} \\
& \times \int_{t_{1}}^{s}\left(\sum_{i=1}^{m}\left|P_{i}(u)\right| M_{3}+|g(u)|\right) d u d s \\
\leq \quad & +c_{3}+1-c_{3}=4
\end{aligned}
$$

and

$$
\begin{array}{rl}
\left(T_{1} x\right)(t)+ & \left(T_{2} y\right)(t) \\
\geq \quad & 3+c_{3}-C(t) x(t-\tau) \\
& -\frac{1}{\Gamma(\alpha+1)} \int_{t}^{\infty} \frac{(s-t)^{\alpha}}{r(s)} \\
& \times \int_{t_{1}}^{s}\left(\sum_{i=1}^{m}\left|P_{i}(u)\right|\left|F_{i}\left(x\left(u-\sigma_{i}\right)\right)\right|\right. \\
\quad+|g(u)|) d u d s & 3+c_{3}-4 c_{3}-\frac{1}{\Gamma(\alpha+1)} \int_{t}^{\infty} \frac{(s-t)^{\alpha}}{r(s)} \\
\geq & \times \int_{t_{1}}^{s}\left(\sum_{i=1}^{m}\left|P_{i}(u)\right| M_{3}+|g(u)|\right) d u d s \\
\geq & 3+c_{3}-4 c_{3}-\left(1-c_{3}\right) \\
= & 2\left(1-c_{3}\right) .
\end{array}
$$

Thus, $\left(T_{1}\right) x(t)+\left(T_{2}\right) y(t) \in \Omega$ for any $x, y \in \Omega$.

Proceeding similarly as in the proof of Theorem 3.1, we obtain that the mapping $T_{1}$ is contraction on $\Omega$ and $T_{2}$ is completely continuous. Thus, by Krasnosel'skii's fixed point theorem, $T_{1} x+T_{2} x=x$ has a solution in $\Omega$, which is a bounded positive solution of Eq.(1.1). Hence the proof is complete.

Theorem 3.4. Assume that $1<c_{4} \equiv C(t)<\infty$ and that conditions (3.1) and (3.2) hold. Then Eq.(1.1) has a bounded nonoscillatory solution.

Proof. Because of conditions (3.1) and (3.2), we can choose a $t_{1}>t_{0}$,

$$
t_{1}+\tau \geq t_{0}+\max \left\{\sigma_{i}\right\}
$$

sufficiently large such that

$$
\begin{aligned}
& \frac{1}{c_{4} \Gamma(\alpha+1)} \int_{t+\tau}^{\infty} \frac{(s-t-\tau)^{\alpha}}{r(s)} \\
& \times \int_{t_{1}+\tau}^{s}\left(\sum_{i=1}^{m}\left|P_{i}(u)\right| M_{4}+|g(u)|\right) d u d s \leq c_{4}-1,
\end{aligned}
$$

where $M_{4}=\max _{2\left(c_{4}-1\right) \leq x \leq 4 c_{4}}\left\{\left|F_{i}(x)\right|: 1 \leq i \leq m\right\}$.

Let $C\left(\left[t_{0}, \infty\right), \mathbb{R}\right)$ be the set of all continuous functions with the norm $\|x\|=\sup _{t \geq t_{0}}|x(t)|<\infty$. Then $C\left(\left[t_{0}, \infty\right), \mathbb{R}\right)$ is a Banach space. We define a closed, bounded and convex subset $\Omega$ of $C\left(\left[t_{0}, \infty\right), \mathbb{R}\right)$ as follows:

$\Omega=\left\{x=x(t) \in C\left(\left[t_{0}, \infty\right), \mathbb{R}\right): 2\left(c_{4}-1\right) \leq x \leq 4 c_{4}, t \geq t_{0}\right\}$

Define two maps $T_{1}$ and $T_{2}: \Omega \rightarrow C\left(\left[t_{0}, \infty\right), \mathbb{R}\right)$ as follows:

$$
\left(T_{1} x\right)(t)= \begin{cases}3 c_{4}+1-\frac{1}{C(t)} x(t+\tau), & t \geq t_{1}, \\ \left(T_{1} x\right)\left(t_{1}\right), & t_{0} \leq t \leq t_{1},\end{cases}
$$




$$
\left(T_{2} x\right)(t)= \begin{cases}\frac{1}{C(t) \Gamma(\alpha+1)} \int_{t+\tau}^{\infty} \frac{(s-t-\tau)^{\alpha}}{r(s)} & \\ \times \int_{t_{1}+\tau}^{s}\left(\sum_{i=1}^{m} P_{i}(u) F_{i}\left(x\left(u-\sigma_{i}\right)\right)\right. & \\ \quad-g(u)) d u d s, & t \geq t_{1}, \\ \left(T_{2} x\right)\left(t_{1}\right), & t_{0} \leq t \leq t_{1} .\end{cases}
$$

For every $x, y \in \Omega$ and $t \geq t_{1}$, we get

$$
\begin{aligned}
&\left(T_{1} x\right)(t)+\left(T_{2} y\right)(t) \\
& \leq 3 c_{4}+1-\frac{1}{C(t)} x(t+\tau) \\
&+\frac{1}{C(t) \Gamma(\alpha+1)} \int_{t+\tau}^{\infty} \frac{(s-t-\tau)^{\alpha}}{r(s)} \\
& \times \int_{t_{1}+\tau}^{s}\left(\sum_{i=1}^{m}\left|P_{i}(u)\right|\left|F_{i}\left(x\left(u-\sigma_{i}\right)\right)\right|\right. \\
&+|g(u)|) d u d s \\
& \leq \quad 3 c_{4}+1+\frac{1}{c_{4} \Gamma(\alpha+1)} \int_{t+\tau}^{\infty} \frac{(s-t-\tau)^{\alpha}}{r(s)} \\
& \times \int_{t_{1}+\tau}^{s}\left(\sum_{i=1}^{m}\left|P_{i}(u)\right| M_{4}+|g(u)|\right) d u d s \\
& \leq \quad 3 c_{4}+1+c_{4}-1=4 c_{4}
\end{aligned}
$$

and

$$
\begin{array}{ll}
\left(T_{1} x\right)(t) & +\left(T_{2} y\right)(t) \\
\geq & 3 c_{4}+1-\frac{1}{C(t)} x(t+\tau) \\
& -\frac{1}{C(t) \Gamma(\alpha+1)} \int_{t+\tau}^{\infty} \frac{(s-t-\tau)^{\alpha}}{r(s)} \\
& \times \int_{t_{1}+\tau}^{s}\left(\sum_{i=1}^{m}\left|P_{i}(u)\right|\left|F_{i}\left(x\left(u-\sigma_{i}\right)\right)\right|+|g(u)|\right) d u d s \\
\geq \quad 3 c_{4}+1-4-\frac{1}{c_{4} \Gamma(\alpha+1)} \int_{t+\tau}^{\infty} \frac{(s-t-\tau)^{\alpha}}{r(s)} & \times \int_{t_{1}+\tau}^{s}\left(\sum_{i=1}^{m}\left|P_{i}(u)\right| M_{4}+|g(u)|\right) d u d s \\
\geq \quad 3 c_{4}+1-\left(c_{4}-1\right)=2\left(c_{4}-1\right) .
\end{array}
$$

Thus, $\left(T_{1}\right) x(t)+\left(T_{2}\right) y(t) \in \Omega$ for any $x, y \in \Omega$.

Proceeding similarly as in the proof of Theorem 3.1, we obtain that the mapping $T_{1}$ is contraction on $\Omega$ and $T_{2}$ is completely continuous. Thus, by Krasnosel'skii's fixed point theorem, $T_{1} x+T_{2} x=x$ has a solution in $\Omega$, which is a bounded positive solution of Eq.(1.1). Hence the proof is complete.

Theorem 3.5. Assume that $C(t) \equiv-1$ and that conditions (3.1) and (3.2) hold. Then Eq.(1.1) has a bounded nonoscillatory solution.

Proof. Because of conditions (3.1) and (3.2), we can choose a $t_{1}>t_{0}$,

$$
t_{1}+\tau \geq t_{0}+\max \left\{\sigma_{i}\right\}
$$

sufficiently large such that

$$
\begin{aligned}
& \frac{1}{\Gamma(\alpha+1)} \sum_{j=1}^{\infty} \int_{t+j \tau}^{\infty} \frac{(s-t-j \tau)^{\alpha}}{r(s)} \\
& \times \int_{t_{1}+j \tau}^{s}\left(\sum_{i=1}^{m}\left|P_{i}(u)\right| M_{5}+|g(u)|\right) d u d s \leq 1,
\end{aligned}
$$

where $M_{5}=\max _{0 \leq x \leq 1}\left\{\left|F_{i}(x)\right|: 1 \leq i \leq m\right\}$.

Let $C\left(\left[t_{0}, \infty\right), \mathbb{R}\right)$ be the set of all continuous functions with the norm $\|x\|=\sup _{t \geq t_{0}}|x(t)|<\infty$. Then $C\left(\left[t_{0}, \infty\right), \mathbb{R}\right)$ is a Banach space. We define a closed, bounded and convex subset $\Omega$ of $C\left(\left[t_{0}, \infty\right), \mathbb{R}\right)$ as follows:

$$
\Omega=\left\{x=x(t) \in C\left(\left[t_{0}, \infty\right), \mathbb{R}\right): 2 \leq x \leq 4, t \geq t_{0}\right\} .
$$

Define a mapping $T: \Omega \rightarrow C\left(\left[t_{0}, \infty\right), \mathbb{R}\right)$ as follows:

$$
(T x)(t)= \begin{cases}3-\frac{1}{\Gamma(\alpha+1)} \sum_{j=1}^{\infty} \int_{t+j \tau}^{\infty} \frac{(s-t-j \tau)^{\alpha}}{r(s)} & \\ \times \int_{t_{1}+j \tau}^{s}\left(\sum_{i=1}^{m} P_{i}(u) F_{i}\left(x\left(u-\sigma_{i}\right)\right)\right. & \\ \quad-g(u)) d u d s, & t \geq t_{1}, \\ (T x)\left(t_{1}\right), & t_{0} \leq t \leq t_{1} .\end{cases}
$$

For every $x \in \Omega$ and $t \geq t_{1}$, we get

$$
\begin{aligned}
(T x)(t) \leq & 3+\frac{1}{\Gamma(\alpha+1)} \sum_{j=1}^{\infty} \int_{t+j \tau}^{\infty} \frac{(s-t-j \tau)^{\alpha}}{r(s)} \\
& \times \int_{t_{1}+j \tau}^{s}\left(\sum_{i=1}^{m}\left|P_{i}(u)\right|\left|F_{i}\left(x\left(u-\sigma_{i}\right)\right)\right|\right. \\
& +|g(u)|) d u d s \\
\leq & 3+\frac{1}{\Gamma(\alpha+1)} \sum_{j=1}^{\infty} \int_{t+j \tau}^{\infty} \frac{(s-t-j \tau)^{\alpha}}{r(s)} \\
& \times \int_{t_{1}+j \tau}^{s}\left(\sum_{i=1}^{m}\left|P_{i}(u)\right| M_{5}+|g(u)|\right) d u d s \\
\leq & 4
\end{aligned}
$$

and

$$
\begin{aligned}
(T x)(t) \geq & 3-\frac{1}{\Gamma(\alpha+1)} \sum_{j=1}^{\infty} \int_{t+j \tau}^{\infty} \frac{(s-t-j \tau)^{\alpha}}{r(s)} \\
& \times \int_{t_{1}+j \tau}^{s}\left(\sum_{i=1}^{m}\left|P_{i}(u)\right|\left|F_{i}\left(x\left(u-\sigma_{i}\right)\right)\right|\right. \\
& +|g(u)|) d u d s \\
\geq & 3-\frac{1}{\Gamma(\alpha+1)} \sum_{j=1}^{\infty} \int_{t+j \tau}^{\infty} \frac{(s-t-j \tau)^{\alpha}}{r(s)} \\
& \times \int_{t_{1}+j \tau}^{s}\left(\sum_{i=1}^{m}\left|P_{i}(u)\right| M_{5}+|g(u)|\right) d u d s \\
\geq & 2 .
\end{aligned}
$$

Thus, $T \Omega \subset \Omega$. Next, we have to show that $T$ is completely continuous. It is enough to show that $T$ is continuous and $T \Omega$ is relatively compact. 
First, we will show that $T$ is continuous. Let $x_{k}(t)$ be a sequence in $\Omega$ such that $x_{k}(t) \rightarrow x(t)$ as $k \rightarrow \infty$. Since $\Omega$ is closed, $x=x(t) \in \Omega$. For $t \geq t_{1}$, we have

$$
\begin{aligned}
& \left|\left(T x_{k}\right)(t)-(T x)(t)\right| \\
& \leq \frac{1}{\Gamma(\alpha+1)} \sum_{j=1}^{\infty} \int_{t+j \tau}^{\infty} \frac{(s-t-j \tau)^{\alpha}}{r(s)} \\
& \quad \times \int_{t_{1}+j \tau}^{s}\left(\sum_{i=1}^{m}\left|P_{i}(u)\right| \mid F_{i}\left(x_{k}\left(s-\sigma_{i}\right)\right)\right. \\
& \left.\quad-F_{i}\left(x\left(s-\sigma_{i}\right)\right) \mid\right) d u d s
\end{aligned}
$$

Since $\left|F_{i}\left(x_{k}\left(t-\sigma_{i}\right)\right)-F_{i}\left(x\left(t-\sigma_{i}\right)\right)\right| \rightarrow 0$ as $k \rightarrow \infty$ for $i=$ $1,2, \ldots, m$, by Lebesgue-dominated convergence theorem, we get that $\lim _{t \rightarrow \infty}\left\|\left(T x_{k}\right)(t)-(T x)(t)\right\|=0$. Thus, $T$ is continuous.

In order to show that $\mathscr{B} \Omega$ is relatively compact, it is enough to show that the family of functions $T x: x \in \Omega$ is uniformly bounded and equicontinuous on $\left[t_{0}, \infty\right)$. The uniformly boundedness is obvious. For the equicontinuity, by Levitan's result [3], it is enough to show that, for any given $\varepsilon>0,\left[t_{1}, \infty\right)$ can be decomposed into finite subintervals in such a way that all functions of the family have oscillation less than $\varepsilon$ on each subinterval. By (3.1) and (3.2), for any $\varepsilon>0$, choose $K \geq t_{1}$ sufficiently large such that

$$
\begin{aligned}
& \frac{1}{\Gamma(\alpha+1)} \sum_{j=1}^{\infty} \int_{T+j \tau}^{\infty} \frac{(s-t-j \tau)^{\alpha}}{r(s)} \\
& \quad \times \int_{t_{1}+j \tau}^{s}\left(\sum_{i=1}^{m}\left|P_{i}(u)\right| M_{5}+|g(u)|\right) d u d s<\frac{\varepsilon}{2} .
\end{aligned}
$$

For $t_{3}>t_{2} \geq K$ and $x \in \Omega$, we obtain

$$
\begin{aligned}
\left|(T x)\left(t_{3}\right)-(T x)\left(t_{2}\right)\right| & \frac{1}{\Gamma(\alpha+1)} \sum_{j=1}^{\infty} \int_{t_{3}+j \tau}^{\infty} \frac{(s-t-j \tau)^{\alpha}}{r(s)} \\
& \times \int_{t_{1}+j \tau}^{s}\left(\sum_{i=1}^{m}\left|P_{i}(u)\right|\left|F_{i}\left(x\left(s-\sigma_{i}\right)\right)\right|+|g(u)|\right) d u d s \\
& +\frac{1}{\Gamma(\alpha+1)} \sum_{j=1}^{\infty} \int_{t_{2}+j \tau}^{\infty} \frac{(s-t-j \tau)^{\alpha}}{r(s)} \\
& \times \int_{t_{1}+j \tau}^{s}\left(\sum_{i=1}^{m}\left|P_{i}(u)\right|\left|F_{i}\left(x\left(s-\sigma_{i}\right)\right)\right|+|g(u)|\right) d u d s \\
\leq \quad & \frac{1}{\Gamma(\alpha+1)} \sum_{j=1}^{\infty} \int_{t_{3}+j \tau}^{\infty} \frac{(s-t-j \tau)^{\alpha}}{r(s)} \\
& \times \int_{t_{1}+j \tau}^{s}\left(\sum_{i=1}^{m}\left|P_{i}(u)\right| M_{5}+|g(u)|\right) d u d s \\
& +\frac{1}{\Gamma(\alpha+1)} \sum_{j=1}^{\infty} \int_{t_{2}+j \tau}^{\infty} \frac{(s-t-j \tau)^{\alpha}}{r(s)} \\
& \times \int_{t_{1}+j \tau}^{s}\left(\sum_{i=1}^{m}\left|P_{i}(u)\right| M_{5}+|g(u)|\right) d u d s
\end{aligned}
$$

$$
<\frac{\varepsilon}{2}+\frac{\varepsilon}{2}=\varepsilon .
$$

For $t_{1} \leq t_{2}<t_{3} \leq K$ and $x \in \Omega$, we choose a sufficiently large $J \in \mathbb{N}$ such that $t_{1}+j \tau \geq K$ if $j \geq J$. For $x \in \Omega$

$$
\begin{aligned}
\left|(T x)\left(t_{3}\right)-(T x)\left(t_{2}\right)\right| & \frac{1}{\Gamma(\alpha+1)} \sum_{j=1}^{\infty} \int_{t_{2}+j \tau}^{t_{3}+j \tau} \frac{(s-t-j \tau)^{\alpha}}{r(s)} \\
& \times \int_{t_{1}+j \tau}^{s}\left(\sum_{i=1}^{m}\left|P_{i}(u)\right|\left|F_{i}\left(x\left(s-\sigma_{i}\right)\right)\right|+|g(u)|\right) d u d s \\
\leq & \frac{1}{\Gamma(\alpha+1)} \sum_{j=1}^{J} \int_{t_{2}+j \tau}^{t_{3}+j \tau} \frac{(s-t-j \tau)^{\alpha}}{r(s)} \\
& \times \int_{t_{1}+j \tau}^{s}\left(\sum_{i=1}^{m}\left|P_{i}(u)\right| M_{5}+|g(u)|\right) d u d s \\
& +\frac{1}{\Gamma(\alpha+1)} \sum_{j=J+1}^{\infty} \int_{t_{2}+j \tau}^{t_{3}+j \tau} \frac{(s-t-j \tau)^{\alpha}}{r(s)} \\
& \times \int_{t_{1}+j \tau}^{s}\left(\sum_{i=1}^{m}\left|P_{i}(u)\right| M_{5}+|g(u)|\right) d u d s \\
\leq \quad & \frac{1}{\Gamma(\alpha+1)} \max _{t_{1}+j \tau \leq s \leq T+j \tau}\left\{\frac{(s-t-j \tau)^{\alpha}}{r(s)}\right. \\
& \left.\times \int_{t_{1}}^{s}\left(\sum_{i=1}^{m}\left|P_{i}(u)\right| M_{5}+|g(u)|\right) d u\right\} J\left(t_{3}-t_{2}\right),
\end{aligned}
$$

and hence there exists a $\delta>0$ such that

$$
\left|(T x)\left(t_{3}\right)-(T x)\left(t_{2}\right)\right|<\varepsilon, \text { whenever } 0<t_{3}-t_{2}<\delta \text {. }
$$

Also, for $t_{0} \leq t_{2}<t_{3} \leq t_{1}$ and $x \in \Omega$, we can easily see that

$$
\left|(T x)\left(t_{3}\right)-(T x)\left(t_{2}\right)\right|=0<\varepsilon .
$$

Therefore, $\{T x: x \in \Omega\}$ is uniformly bounded and equicontinuous on $\left[t_{0}, \infty\right)$ and hence $T \Omega$ is relatively compact. Thus, by Schauder's fixed point theorem, $T x=x$ has a solution in $\Omega$, which is a bounded positive solution of Eq.(1.1). Hence the proof is complete.

\section{Acknowledgements}

This work is partially supported by the DST-FIST Scheme (Grant No: SR/FST/MSI-115/2016), New Delhi, India.

\section{References}

[1] R. P. Agarwal, S. R. Grace and D. O'Regan; Oscillation Theory for Second Order Linear, Half-Linear, Superlinear and Sublinear Dynamic Equations, Kluwer Academic, Dordrecht, 2002.

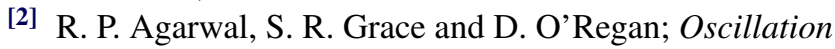
Theory for Second Order Dynamic Equations, Taylor and Francis, London and New York, 2003. 
${ }^{[3]}$ R. P. Agarwal, M. Bohner and W. T. Li; Nonoscillation and Oscillation: Theory for Functional Differential Equations, Dekker, New York, 2004.

[4] D. Chen; Oscillation criteria of fractional differential equations, Adv. Differ. Equ., 33(2012), 1-10.

[5] Q. Feng and F. Meng; Oscillation of solutions to nonlinear forced fractional differential equations, Electr. J. Differ. Equ., 169(2013), 1-10.

[6] S. R. Grace, R. P. Agarwal, P. J. Y. Wong and A. Zafer; On the oscillation of fractional differential equations, Fract. Calc. Appl. Anal., 15(2012), 222-231.

[7] A. A. Kilbas, H. M. Srivastava and J. J. Trujillo; Theory and Applications of Fractional Differential Equations, Elsevier, Amsterdam, 2006.

${ }^{[8]}$ K. S. Miller and B. Ross; An Introduction to the Fractional Calculus and Fractional Differential Equations, Wiley, New York, 1993.

[9] Y. Pan and R. Xu; Some new oscillation criteria for a class of nonlinear fractional differential equations, Fractional Differ. Calc., 6(2016), 17-33.

[10] I. Podlubny; Fractional Differential Equations, Academic Press, San Diego, 1999.

[11] Y. Zhou, B. Ahmed and A. Alsaedi; Existence of nonoscillatory solutions for fractional neutral differential equations, Appl. Math. Lett., 72(2017), 70-74.

[12] Y. Zhou, B. Ahmed and A. Alsaedi; Existence of nonoscillatory solutions for fractional functional differential equations, B. Malays. Math. Sci. So., 2017(2017), 1-16.

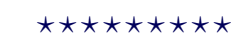

ISSN(P):2319- 3786

Malaya Journal of Matematik

ISSN(O):2321 - 5666

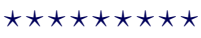

